\title{
Nonexpansive retracts and weak compatible pairs in metric Spaces
}

N Hussain ${ }^{1 *}, G$ Jungck $^{2}$ and MA Khamsi, ${ }^{3,4}$

\footnotetext{
* Correspondence: nhusain@kau. edu.sa

${ }^{1}$ Department of Mathematics, King Abdulaziz University, P.O. Box 80203, Jeddah 21589, Saudi Arabia Full list of author information is available at the end of the article
}

\begin{abstract}
In this article, we introduce a new approach to common fixed point theory for a weak compatible pair. We first introduce the concepts of R-pair and NR-pair and establish some new common fixed point theorems for a weak compatible pair in hyperconvex metric spaces and uniformly convex metric spaces. We shall also establish the well-known De Marr's theorem for a family of weak compatible pairs in a hyperconvex metric space $[0,1]$ and show by an example that it fails to hold in general hyperconvex metric spaces.

Mathematics Subject Classification (2000): Primary 06F30, 46B20, 47E10.
\end{abstract}

Keywords: hyperconvex metric space, uniformly convex metric space, continuous retraction, nonexpansive mapping, weakly compatible pair

\section{Introduction}

The celebrated result on the existence of a common fixed point for nonexpansive commutative family was first established by DeMarr [1] under the assumption that $C$ is a compact convex subset of a normed space $X$. In 1992, Khamsi et al. [2] established above-mentioned results for a finite as well as an arbitrary commutative family of maps in hyperconvex metric spaces. More recently, Hussain et al. [3] have extended the results in [2] to the family of symmetric Banach operator pairs. In this article, we first introduce the concepts of $R$-pair and $N R$-pair and establish some new common fixed point theorems for a weak compatible pair in hyperconvex metric spaces and uniformly convex metric spaces. We shall also establish the well-known De Marr's theorem for a family of weak compatible pairs in a special hyperconvex metric space.

\section{Basic definitions and results}

A metric space $H$ is said to be hyperconvex [4] if given any family $\left\{x_{\alpha}\right\}$ of points of $H$ and any family $\left\{r_{\alpha}\right\}$ of nonnegative real numbers satisfying

$$
d\left(x_{\alpha}, x_{\beta}\right) \leq r_{\alpha}+r_{\beta}
$$

it is the case that $\cap_{\alpha} B\left(x_{\alpha} ; r_{\alpha}\right) \neq \emptyset$.

Definition 2.1. The ordered pair $(S, T)$ of two self-maps of a metric space $H$ is called a Banach operator pair, if the set $\operatorname{Fix}(T)$ is $S$-invariant, namely $S(\operatorname{Fix}(T)) \subseteq \operatorname{Fix}(T)$.

Obviously a commuting pair $(S, T)$ is a Banach operator pair but not conversely in general, see [5-8].

(c) 2012 Hussain et al; licensee Springer. This is an Open Access article distributed under the terms of the Creative Commons Attribution License (http://creativecommons.org/licenses/by/2.0), which permits unrestricted use, distribution, and reproduction in any medium, provided the original work is properly cited. 
Definition 2.2. Let $T$ and $S$ be two self-maps of a metric space $H$. The pair $(S, T)$ is called symmetric Banach operator pair if both $(S, T)$ and $(T, S)$ are Banach operator pairs, i.e., $T(\operatorname{Fix}(S)) \subseteq \operatorname{Fix}(S)$ and $S(\operatorname{Fix}(T)) \subseteq \operatorname{Fix}(T)$.

It is easy to see that the pair $(S, T)$ is a symmetric Banach operator pair if and only if $T$ and $S$ are commuting on $\operatorname{Fix}(T) \cup \operatorname{Fix}(S)$.

Definition 2.3. Let $X$ be a metric space and $S, T$ self-maps of $X$. A point $x$ is called a coincidence point of $S$ and $T$ iff $S x=T x$. We shall call $w=S x=T x$ a point of coincidence of $S$ and $T$. Let $C(S, T)=\{x: S x=T x\}$ and $\operatorname{PC}(S, T)=\{w: \exists x \in X$, with $w=$ $S x=T x\}$ denote the sets of coincidence points and points of coincidence, respectively, of the pair $(S, T)$. Clearly, $\operatorname{PC}(S, T)=T(C(S, T))=S(C(S, T))$. The maps $S$ and $T$ are called weakly compatible $[9,10]$ if they commute at their coincidence points, i.e., $S T x=$ $T S x$ for each $x$ in $C(S, T)$; equivalently, $S$ and $T$ are weakly compatible if the set $C(S$, $T)$ is $S$ - and $T$-invariant, namely, $S(C(S, T)) \subseteq C(S, T)$ and $T(C(S, T)) \subseteq C(S, T)$. We say $S$ and $T$ are nontrivially weakly compatible if and only if they are weakly compatible and $C(S, T) \neq \varphi$. The maps $S$ and $T$ are called compatible if $\lim _{n} d\left(T S x_{n}, S T x_{n}\right)=0$ whenever $\left\{x_{n}\right\}$ is a sequence such that $\lim _{n} T x_{n}=\lim _{n} S x_{n}=t$ for some $t$ in $X$.

Example 2.1. Let $S, T:[0,1] \rightarrow[0,1]$ be defined by $T(x)=1-x, S(x)=1-x^{2}$ on $[0,1]$. Then the pair $(S, T)$ is weakly compatible (in fact, compatible), but not a Banach operator pair.

Example 2.2. Let $S, T:[0,1] \rightarrow[0,1]$ be defined by $T(x)=(1+x) / 2, S(x)=(1+2 x-$ $\left.x^{2}\right) / 2$ on $[0,1]$. Then the pair $(S, T)$ is a symmetric Banach operator pair but not weakly compatible.

Example 2.3. Note that $[0,1]$ is a compact hyperconvex metric space. Let $T:[0,1] \rightarrow$ $[0,1]$ and $S:[0,1] \rightarrow[0,1]$ defined as

$$
T(x)=x^{2} \quad \text { and } \quad S(x)=2 x-x^{2} .
$$

Then $T$ and $S$ do not commute but since $T$ and $S$ commute on $C(S, T)=\{0,1\}=$ PC $(S, T)$, so $T$ and $S$ are weakly compatible. It is worth mentioning that $\mathrm{PC}(S, T)$ is not a continuous retract of $[0,1]$.

Definition 2.4. A geodesic metric space $(M, d)$ is said to be uniformly convex if for any $r>0$ and any $\varepsilon \in(0,2]$ there exists $\delta \in(0,1]$ such that for all $a, x, y \in M$ with $d$ $(x, a) \leq r, d(y, a) \leq r$ and $d(x, y) \geq \varepsilon r$ it is the case that

$$
d(m, a) \leq(1-\delta) r,
$$

where $m$ stands for any midpoint of any geodesic segment $[x, y]$. A mapping $\delta:(0$, $+\infty) \times(0,2] \rightarrow(0,1]$ providing such a $\delta=\delta(r, \varepsilon)$ for a given $r>0$ and $\varepsilon \in(0,2]$ is called a modulus of uniform convexity.

Notice that this definition of uniform convex metric spaces is weaker than the one used in [11] in two ways. First, we do not impose that the metric is convex and, second, our modulus of convexity does depend on the two variables $r$ and $\varepsilon$ while it is assumed to depend only on $\varepsilon$ in [11].

Definition 2.5. Let $(M, d)$ be a metric space, then the metric is said to be convex if for any $x, y$ and $z$ in $M$, and $m$ a midpoint in between $x$ and $y$,

$$
d(z, m) \leq 1 / 2(d(z, x)+d(z, y)) .
$$


It is easy to see that uniformly convex metric spaces are uniquely geodesic, that is, for each two points there is just one geodesic joining them. Therefore, midpoints and geodesic segments $[x, y]$ joining two points are unique. In this case, there is a natural way to define convexity. A subset $C$ of a (uniquely) geodesic space is said to be convex if $[x, y] \subseteq C$ for any $x, y \in C$. For more about geodesic spaces the reader may check [12].

To obtain our results we will need to impose additional conditions on the modulus of convexity (see $[12,13])$.

Definition 2.6. If a uniformly convex metric space $M$ admits a modulus of convexity $\delta$ such that it decreases with $r$ (for each fixed $\varepsilon$ ) then we say $\delta$ is a monotone modulus of convexity for $M$.

In the same way, we define a lower semicontinuous from the right modulus of convexity as follows.

Definition 2.7. If a uniformly convex metric space $M$ admits a modulus of convexity $\delta$ such that it is lower semicontinuous from the right with respect to $r$ (for each fixed $\varepsilon)$ then we say $\delta$ is a lower semicontinuous from the right modulus of convexity for $X$.

Definition 2.8. [13,14] Let $(M, d)$ be a metric space and $f: M \rightarrow M$. Then $f$ satisfies condition $(C)$ if, $1 / 2 d(x, f(x)) \leq d(x, y) \Rightarrow d(f(x), f(y)) \leq d(x, y)$, for all $x, y \in M$; condition $(D)$ if, $1 / 2 d(x, f(x)) \geq d(x, y) \Rightarrow d(f(x), f(y)) \leq d(x, y)$, for all $x, y \in M$.

Obviously, every nonexpansive mapping meets condition $(C)$. Any 2-Lipschitz mapping satisfies condition $(D)$. Notice also that these conditions do not imply continuity and that condition $(D)$ is implied by condition $(C)$ for $x, y$ such that $(1 / 2) d(x, T(x))=d(x, y)$.

Definition 2.9. Let $(M, d)$ be a metric space. $T: M \rightarrow M$ will be said to be an asymptotic pointwise nonexpansive mapping if there exists a sequence of mappings $\alpha_{n}$ $: M \rightarrow[0, \infty)$ such that

$$
d\left(T^{n}(x), T^{n}(y)\right) \leq \alpha_{n}(x) d(x, y)
$$

and

$$
\limsup _{n \rightarrow \infty} \alpha_{n}(x) \leq 1
$$

for any $x, y \in M$. For more details on it we refer [15].

\section{Common fixed points for weak compatible pairs}

The systematic study of a common fixed point of a pair of commuting mappings in the setting of complete metric spaces was initiated by Jungck [16], which yields a generalization of the Banach contraction principle as a corollary. Since then, many fixed point theorists have attempted to find weaker forms of commutativity that may ensure the existence of a common fixed point for a pair of self-mappings on a metric space. In this context, the notions of weakly compatible mappings [10] and Banach operator pairs [5,7] have been of significant interest for generalizing results in metric fixed point theory for single valued mappings.

We shall need the following fixed point result.

Lemma 3.1. [3] Let $H$ be a hyperconvex metric space. Let $T: H \rightarrow H$ be continuous such that $\overline{T(H)}$ is compact. Then there exists $K \subset H$ compact hyperconvex such that $T$ $(K) \subset K$. Moreover Fix $(T)$ is not empty and compact. 
We know that if $S$ and $T$ are weakly compatible and have a unique point of coincidence $w=S x=T x$, then $w$ is the unique common fixed point of $S$ and $T$ (see Proposition 1.4 in [17]). In this instance, if $S$ or $T$ is injective, we have $\operatorname{PC}(S, T)=C(S, T)$, since $x=w$. However, even though $S$ and $T$ are continuous, weakly compatible, and have a unique point of coincidence and therefore a unique common fixed point, $S$ and $T$ may have more than one coincident point. For this we consider the following example.

Example 3.1. Let $S(x)=1$ and $T(x)=4\left(x-\frac{1}{2}\right)^{2}$ for all $x \in[0,1]$. Then 1 is the unique point of coincidence, but 1 and 0 are coincidence points; i.e., $C(S, T)=\{0,1\}$, whereas $\operatorname{PC}(S, T)=S(C(S, T))=T(C(S, T))=\{1\}$.

The above example suggests the following definition.

Definition 3.1. The pair $S, T: H \rightarrow H$ is called an $R$-pair if $P C(S, T)$ is a continuous retract of $H$.

Example 3.2. Let $M$ be a nonempty subset of a metric space $(X, d)$, and $S$ and $T$ be self-maps of $M$. Assume that $\operatorname{cl}(T(M)) \subset S(M), \operatorname{cl}(T(M))$ is complete, and $S$ and $T$ satisfy for all $x, y \in M$ and for some $k, 0 \leq k<1$,

$$
d(T x, T y) \leq k \max \{d(S x, S y), d(T x, S x), d(T y, S y), d(T x, S y), d(T y, S x)\} .
$$

Then by Corollary 2.2 in [18], $\mathrm{PC}(S, T)$ is a singleton and hence a continuous retract of $M$. Thus the pair $(S, T)$ is an $R$-pair.

Note that the set of coincidence points and the set of points of coincidence of two continuous functions defined on any compact hyperconvex metric space may not be continuous retract (see Example 2.3).

Theorem 3.1. Let $H$ be a hyperconvex metric space and let $S$ and $T$ be continuous self-maps of $H$ such that $\overline{P C(S, T)}$ is compact. If $(S, T)$ is an R-pair which is weakly compatible, then $S$ and $T$ have a common fixed point.

Proof. Since $S$ and $T$ are weakly compatible, (i) $S(\mathrm{PC}(S, T)) \subset \mathrm{PC}(S, T) \subset C(S, T)$. Moreover, $(S, T)$ is an $R$-pair, so there is a continuous retract $R: H \rightarrow \operatorname{PC}(S, T)$ such that $(i i) R(x)=x$ for $x \in \operatorname{PC}(S, T)$. Thus, $\operatorname{PC}(S, T)$ is not empty and $S \circ R: H \rightarrow H$ is a continuous map such that $S \circ R(H)=S(R(H)) \subset S(\mathrm{PC}(S, T))$. Therefore, $(i)$ implies that (iii) $\overline{S \circ R(H)} \subset \overline{\mathrm{PC}(S, T)} \subset C(S, T)$, since $S$ and $T$ are continuous and $C(S, T)$ is thus closed. But $\overline{\mathrm{PC}(S, T)}$ is compact by hypothesis, so that $\overline{S \circ R(H)}$ is compact by (iii). Lemma 3.1 thus implies that $S \circ R$ has a fixed point $z$ in $\operatorname{PC}(S, T)$; i.e., $(S \circ R)(z)$ $=S(R(z))=S(z)=z$, by $(i i)$. Since $z$ is in $C(S, T)$ by $(i), T(z)=S(z)=z$, as desired.

Corollary 3.1. Let $H$ be a hyperconvex metric space and $S, T: H \rightarrow H$ be a continuous R-pair such that $\overline{T(H)}$ is compact. If $(S, T)$ is a weakly compatible pair, then Fix $(S)$ $\cap \operatorname{Fix}(T)$ is not empty.

Proof. Since the pair $(S, T)$ is weakly compatible, so $\overline{\operatorname{PC}(S, T)}=\overline{T(C(S, T))} \subset \overline{T(H)}$ which implies that $\overline{\mathrm{PC}(S, T)}$ is compact. The result now follows from Theorem 3.1.

Corollary 3.2. Let $H$ be a compact hyperconvex metric space. Let $S, T: H \rightarrow H$ be a continuous R-pair. If $(S, T)$ is a weakly compatible pair, then $\operatorname{Fix}(S) \cap \operatorname{Fix}(T)$ is not empty.

Theorem 3.2. Let $H$ be a hyperconvex metric space and $S, T: H \rightarrow H$ be a weak compatible pair. Suppose that $P C(S, T)$ is a nonempty bounded and hyperconvex subset 
of $H$. If $S: H \rightarrow H$ satisfies condition $(C)$ and $(D)$, then $S$ and $T$ have a common fixed point.

Proof. Since $S$ and $T$ are weakly compatible, $S(\mathrm{PC}(S, T)) \subset \mathrm{PC}(S, T) \subset C(S, T)$. Also $S$ satisfies condition $(C)$ and $(D)$ on $\operatorname{PC}(S, T)$ and $S$ maps $\mathrm{PC}(S, T)$ into itself which is bounded and hyperconvex. Thus, by Theorem 3.23 [13], $S$ has a fixed point $z$ in $\operatorname{PC}(S$, $T)$. As $P C(S, T) \subset C(S, T)$, so $z$ is in $C(S, T)$. Thus $T(z)=S(z)=z$, as desired.

Example 3.3. Let $T, S:[0,1] \rightarrow[0,1]$ be defined as

$$
T(x)=1-x \quad \text { and } \quad S(x)=(1-x)^{2} .
$$

Then $T$ and $S$ commute on $C(S, T)=\{0,1\}=\mathrm{PC}(S, T)$, so $T$ and $S$ are weakly compatible. All the conditions of Theorem 3.1 are satisfied except the pair $(S, T)$ is an $R$ pair and $T$ and $S$ have no common fixed point.

Example 3.4. Let $T, S:[0,1] \rightarrow[0,1]$ be defined as

$$
T(x)=1 \quad \text { and } \quad S(x)=1-x .
$$

Then $T$ and $S$ satisfy all aspects of the hypothesis of Theorem 3.1 except weak compatibility. Note that $C(S, T)=\{0\}, \operatorname{PC}(S, T)=\{1\}$ and $T S 0=1 \neq 0=S T 0$ and $T$ and $S$ have no common fixed point. We also notice here that $C(S, T)=\{0\}$ is not a subset of $\overline{T([0,1])}$

If $S, T$ are continuous functions on a metric space $X$, then $C(S, T)$ is obviously a closed subset of $X$. We provide an example of continuous functions $S, T: Y \rightarrow Y$ with $Y$ closed in $X, S(Y)=T(Y)$ compact, but $\mathrm{PC}(S, T)$ is not closed.

Example 3.5. Let $X=\{-2,-1\} \cup[0, \infty), S(-2)=T(-1)=1, S(-1)=T(-2)=0, S(x)=T$ $(x)=x(1+x)^{-1}$ for all $x \in[0, \infty)$. Then $\mathrm{PC}(S, T)=[0,1)$, not closed in $X$.

Recall that $A \subset X$ is a nonexpansive retract of $X$ if there exists a nonexpansive map $R$ $: X \rightarrow A$ such that $R(a)=a$ for every $a \in A$.

Definition 3.2. The pair $S, T: M \rightarrow M$ is called NR-pair if $\mathrm{PC}(S, T)$ is a nonexpansive retract of $M$.

It is well-known that if $H$ is a bounded hyperconvex metric space and $T: H \rightarrow H$ is nonexpansive, then $\operatorname{Fix}(T)$ is hyperconvex and consequently is a nonexpansive retract of $H$. Note that if $S, T$ are both nonexpansive and $H$ is a bounded hyperconvex metric space, then $C(S, T)$ as well as $\operatorname{PC}(S, T)$ need not be hyperconvex.

Example 3.6. Consider $\mathbb{R}^{2}$ with the supremum norm, $M$ as the square, with its interior, of vertices $(1,1),(1,-1),(-1,1)$ and $(-1,-1)$ and with the induced metric. Let

$$
X_{1}=\{(x, y): x \in[-1,1] \text { and } y=1+x \text { if } x \in[-1,0] \text { and } y=1-x \text { otherwise }\}
$$

and

$$
X_{2}=\left\{(x, y):(x,-y) \in X_{1}\right\}
$$

Define $S: M \rightarrow X_{1}$ and $T: M \rightarrow X_{2}$ to be nonexpansive projections as follows. Let $S$ (resp. $T$ ) send each vertical segment in $M$ into its intersection with $X_{1}$ (resp. $X_{2}$ ). Then $C(S, T)$ would coincide with the vertical segments of $M$ passing through $(-1,0)$ and $(1$, $0)$, and $\operatorname{PC}(S, T)=\{(-1,0),(1,0)\}$. Note that both $X_{1}$ and $X_{2}$ are hyperconvex, but neither $C(S, T)$ nor $\operatorname{PC}(S, T)$ is hyperconvex. Moreover, $S$ and $T$ are weakly compatible. To see this, first note that the points $(-1,0)$ and $(1,0)$ of $\mathrm{PC}(S, T)$ are also common fixed points of $S$ and $T$. Therefore, if $y$ is a point of coincidence of $S$ and $T, y=S y=$ 
$T y$. Now suppose $S x=T x$. Then $S x=T x=y$ for some $y$ in $\mathrm{PC}(S, T)$, and we have $S x=$ $T x=y=S y=T y$. Therefore, $T S x=T y=y$, and $S T x=S y=y$; i.e., $S T x=T S x$, and $S$ and $T$ are weakly compatible.

Throughout this article, we will denote a uniformly convex metric space with monotone (or lower semi-continuous from the right) modulus of uniform convexity as a UC space(see for details $[12,13,19,20]$ ).

We shall need the following results;

Lemma 3.2. [12] Let $X$ be a complete UC space. Suppose $X$ is bounded, then any nonexpansive mapping $T: X \rightarrow X$ has a fixed point.

Lemma 3.3. [13] Let $X$ be a complete UC space with convex metric and suppose $K$ is a nonempty bounded closed convex subset of $X$. If $f: K \rightarrow K$ is nonexpansive, then Fix (f) is nonempty, closed and convex.

Lemma 3.4. ([13], Theorem 2.6) Let $X$ be a complete UC space with convex metric and suppose $K$ is a nonempty bounded closed convex subset of $X$. If $f: K \rightarrow K$ satisfies condition (C), then Fix(f) is nonempty, closed and convex.

Theorem 3.3. Let $M$ be a bounded complete UC space. Let $S, T: M \rightarrow M$ be a NRpair. Let $S: M \rightarrow M$ be nonexpansive such that $(S, T)$ be a weakly compatible pair. Then $S$ and $T$ have a common fixed point.

Proof. Since the retract of a nonempty space is nonempty, $\mathrm{PC}(S, T)$ is nonempty. Since $S$ and $T$ are weakly compatible, $S(\mathrm{PC}(S, T)) \subset \mathrm{PC}(S, T) \subset C(S, T)$. Since $(S, T)$ is an NR-pair, then there exists a nonexpansive retract $R: M \rightarrow P C(S, T)$. Hence $S \circ R$ : $M \rightarrow M$ is nonexpansive map such that $S \circ R(M) \subset P C(S, T)$. Lemma 3.2 implies that $S \circ R$ has a fixed point $z$ in $\operatorname{PC}(S, T)$; i.e., $(S \circ R)(z)=S(R(z))=S(z)=z$. As $\operatorname{PC}(S, T) \subset$ $C(S, T)$, so $z$ is in $C(S, T)$. Thus, $T(z)=S(z)=z$, as desired.

Theorem 3.4. Let $M$ be a complete UC space with convex metric and $S, T$ be a NRpair on a closed bounded convex subset $C$ of $M$. Let $S: C \rightarrow C$ be nonexpansive such that $(S, T)$ be a weakly compatible pair. Then $S$ and $T$ have a common fixed point.

Proof. Proof is similar to that of Theorem 3.3, instead of using Lemma 3.2 we use Lemma 3.3 here.

Fixed point theory in CAT(0) spaces was first studied by Kirk [21]. He showed that every nonexpansive (single-valued) mapping defined on a bounded closed convex subset of a complete CAT(0) space always has a fixed point. Utilizing this observation and proof of Theorem 3.3, we obtain the following result.

Theorem 3.5. Let $S, T$ be a NR-pair on a closed bounded convex subset $C$ of a complete CAT (0) space $M$. Let $S: C \rightarrow C$ be nonexpansive such that $(S, T)$ be a weakly compatible pair. Then $S$ and $T$ have a common fixed point.

Theorem 3.6. Let $M$ be a complete UC space with convex metric and $S, T: M \rightarrow M$ be a weak compatible pair. Suppose that $P C(S, T)$ is a nonempty closed bounded and convex subset of $M$. If $S: M \rightarrow M$ satisfies condition $(C)$, then $S$ and $T$ have a common fixed point.

Proof. Since $S$ and $T$ are weakly compatible, $S(\mathrm{PC}(S, T)) \subset \mathrm{PC}(S, T) \subset C(S, T)$. Also $S$ satisfies condition $(C)$ on $\mathrm{PC}(S, T)$ and $S$ maps $\mathrm{PC}(S, T)$ into itself which is closed bounded and convex. Thus by Lemma 3.4, $S$ has a fixed point $z$ in $\operatorname{PC}(S, T)$. As $\operatorname{PC}(S$, $T) \subset C(S, T)$, so $z$ is in $C(S, T)$. Thus, $T(z)=S(z)=z$, as desired. 
Corollary 3.3. Let $M$ be a complete $C A T(0)$ space and $S, T: M \rightarrow M$ be a weak compatible pair. Suppose that $P C(S, T)$ is a nonempty closed bounded and convex subset of $M$. If $S: M \rightarrow M$ satisfies condition (C), then $S$ and $T$ have a common fixed point.

Notice that the classes of asymptotic pointwise nonexpansive mappings and the maps satisfying condition $(C)$ contain properly the class of nonexpansive mappings. Hussain and Khamsi [15], proved that if $M$ is a complete CAT(0) space and $K$ is a bounded closed nonempty convex subset of $M$, then any $T: K \rightarrow K$ pointwise asymptotically nonexpansive has a fixed point. Utilizing this fact in the proof of Theorem 3.6, we obtain the following result.

Theorem 3.7. Let $M$ be a complete $C A T(0)$ space and $S, T: M \rightarrow M$ be a weak compatible pair. Suppose that $P C(S, T)$ is a nonempty closed bounded and convex subset of $M$. If $S: M \rightarrow M$ is pointwise asymptotically nonexpansive, then $S$ and $T$ have a common fixed point.

Definition 3.3. [22] A mapping $T: N \rightarrow M$ between two metric spaces is said to be a universal nonexpansive map if $T$ is nonexpansive and if given any nonexpansive mapping $S: N \rightarrow M$ there exists $x \in N$ such that $S(x)=T(x)$, i.e. $C(S, T)$ and hence $\operatorname{PC}(S$, $T)$ is nonempty.

We shall need the following result of Kirk [22].

Theorem 3.8. Suppose $M$ is a compact subset of a metric space $N$ and suppose $f: N$ $\rightarrow M$ is a universal nonexpansive map for which $f(M)=M$. Then $M$ is a nonexpansive retract of $N$.

Theorem 3.9. Let $M$ be a bounded and complete UC space. Let $S: M \rightarrow M$ be nonexpansive and $T: M \rightarrow P C(S, T)$ be universal nonexpansive map such that $P C(S, T) \subset$ $T(P C(S, T))$. Assume that $S, T: M \rightarrow M$ be weakly compatible pair such that $P C(S, T)$ is compact. Then Fix $(S) \cap \operatorname{Fix}(T)$ is not empty.

Proof. As $(S, T)$ is a nontrivially weak compatible pair, so $\mathrm{PC}(S, T)$ is nonempty and $\mathrm{PC}(S, T) \subset C(S, T)$ which implies $T(\mathrm{PC}(S, T)) \subset T(C(S, T))=\mathrm{PC}(S, T)$.

Hence, $\mathrm{PC}(S, T)=T(P C(S, T))$. Thus by Theorem 3.8, $\mathrm{PC}(S, T)$ is a nonexpansive retract of $M$ and hence the pair $(S, T)$ is an NR-pair. Now the conclusion follows from Theorem 3.3.

Corollary 3.4. Let $M$ be a bounded and complete UC space. Let $T: M \rightarrow M$ be universal nonexpansive map such that $T(M)$ is compact. Assume that $S: M \rightarrow M$ is nonexpansive such that $T(M) \subset P C(S, T) \subset T(P C(S, T))$. If $(S, T)$ is a weak compatible pair. Then $\operatorname{Fix}(S) \cap \operatorname{Fix}(T)$ is not empty.

Proof. As $T$ is universal nonexpansive and $S$ is nonexpansive, so $C(S, T)$ and hence $\operatorname{PC}(S, T)$ are nonempty. Since $(S, T)$ is a weak compatible pair, $\operatorname{PC}(S, T) \subset C(S, T)$, which implies $T(\mathrm{PC}(S, T)) \subset T(C(S, T))=\mathrm{PC}(S, T)$. Thus, $\mathrm{PC}(S, T)=T(\mathrm{PC}(S, T))$. As $T$ being universal mapping is surjective, $M=T(M) \subset \mathrm{PC}(S, T)=T(C(S, T)) \subset T(M)=$ $M$ and hence $\operatorname{PC}(S, T)$ is compact subset of $M$. Now the conclusion follows from Theorem 3.9.

Corollary 3.5. Under the same conditions of Theorem 3.9 (or Corollary 3.4), if the condition $P C(S, T) \subset T(P C(S, T))$ is replaced with $C(S, T) \subset P C(S, T)$, then the same conclusion follows.

Notice that in Example 3.3, $\mathrm{PC}(S, T)=C(S, T)$. We now give an example of continuous self-maps $S$ and $T$ of the unit interval $I=[0,1]$ which are injective, surjective in 
fact, homeomorphisms, universal, and weakly compatible for which $\operatorname{PC}(S, T)$ is a proper subset of $C(S, T)$.

Example 3.7. Let $T(x)=x^{1 / 2}$ for $x$ in [0,1]. To construct $S$, let $a$ be an interior point of $[0,1]$; i.e., $0<a<1$. Let $S$ be the piecewise linear function determined by the set $\{0$, $\left.a, T a, T^{2} a, \ldots, T^{n} a, \ldots, 1\right\}$. Thus the graph of $S$ consists of the union of the straight line segments connecting $(0,0),(a, T a), \ldots,\left(T^{n-1} a, T^{n} a\right), \ldots,(1,1)$. Therefore, $C(S, T)=\{0$, $\left.a, T a=S a, T^{2} a=S^{2} a, \ldots, T^{n} a=S^{n} a, \ldots, 1\right\}$ and $\operatorname{PC}(S, T)=T(C(S, T))=C(S, T) \backslash\{a\}$.

\section{Common fixed point of weak compatible family}

The aim of this section is to further study the class of weakly compatible maps which properly contains the class of commuting and compatible maps and is different from the class of symmetric Banach operator pairs [3]. We will show by an example that De Marr's result for the weakly compatible family does not hold in the setting of general hyperconvex metric space.

Let $H$ be a hyperconvex metric space and $\mathcal{T}$ be a family of mappings defined on $H$. Then the family $\mathcal{T}$ has a common fixed point if it is the fixed point of each member of $\mathcal{T}$. Hussain et al. [3] proved the following De Marr's result for symmetric Banach operator family in the setting of hyperconvex metric space without the compactness of the domain.

Theorem 4.1. Let $H$ be a hyperconvex metric space. Let $\mathcal{T}$ be a family of nonexpansive mappings defined on $H$. Assume any two mappings from $\mathcal{T}$ form a symmetric Banach operator pair. Then the family $\mathcal{T}$ has a common fixed point provided one map from $\mathcal{T}$ has a bounded nonempty fixed point set. Moreover the common fixed point set $\operatorname{Fix}(\mathcal{T})$ is hyperconvex.

For the remaining portion of this article, we will use the following notation. $\mathbb{N}$ will denote the set of positive integers, and $\mathbb{N}_{n}$ the set of all $k \in \mathrm{N}$ such that $k \leq n$. If $g$ is a self-map of a set $X, P(g)$ will denote the set of periodic points of $g$. Notice that $x$ is a periodic point of $g$ if $g^{k} x=x$ for some $k \in \mathbb{N}$. Also note that in a compact metric space, the concepts of "compatible" and "weakly compatible" are equivalent [9]. Further note that $\operatorname{Fix}(g)$ is a nonempty closed interval if $g$ is a nonexpansive self-map of $[0,1]$.

We shall need the following result.

Theorem 4.2. [23]A continuous self-map $g$ of $[0,1]$ has a common fixed point with each self-map $f$ of $[0,1]$ which is nontrivially compatible with $g$ iff $g$ has no nontrivial periodic points (i.e., $P(g)=$ Fix $(g)$ ).

Regarding our desire to obtain a result analogous to above Theorem 4.1, we have the following partial result in this direction.

Theorem 4.3. Let $\mathcal{F}$ be a family of nonexpansive self-maps of $[0,1]$ which have no nontrivial periodic points. Suppose that any pair $\{f, g\}$ in $\mathcal{F}$ is nontrivially weakly compatible. Then the family $\mathcal{F}$ has a common fixed point. In fact, the set of common fixed points of $\mathcal{F}$ is a closed interval.

Proof. We first consider the finite case. For $n \in \mathbb{N}$, let $P(n)$ denote, "Any subset of $\mathcal{F}$ containing $n$ functions has a common fixed point." $P(1)$ is immediate, and $P(2)$ follows from Theorem 4.2 above. Now let $n$ be an element of $\mathbb{N}$ such that $P(n)$ is true, and suppose that $P(n+1)$ is false. (Note that by the above, $n+1 \geq 3$.) Then there is a subset $G$ of $\mathcal{F}$ having $n+1$ functions $f_{i}$ such that $G$ has no common fixed point. Therefore, since $P(n)$ is true, for each $i$ in $N_{n+1}$, we can let $c_{i}$ be a common fixed 
point of the subset of $G$ obtained by deleting $f_{i}$. Thus, $f_{k}\left(c_{i}\right)=c_{i}$ if and only if $i \neq k$. Clearly, all the $c_{i}$ are distinct. For if there exist $i, k(i \neq k)$ such that $c_{i}=c_{k}$, we have the contradiction: $f_{k}\left(c_{k}\right)=f_{k}\left(c_{i}\right)=c_{i}=c_{k}$. Consequently, since $n+1 \geq 3$ we have distinct $i$, $k, j$ such that $c_{i}<c_{k}<c_{j}$, so that $f_{k}(c i)=c_{i}$ and $f_{k}\left(c_{j}\right)=c_{j}$. But then $\left[c_{i}, c_{j}\right]$ is a subset of $\operatorname{Fix}\left(f_{k}\right)$ since $f_{k}$ is nonexpansive and $\operatorname{Fix}\left(f_{k}\right)$ is an interval; therefore, $f_{k}\left(c_{k}\right)=c_{k}$ a contradiction.

We have proved that the family $\{\operatorname{Fix}(f): f \in \mathcal{F}\}$ has the finite intersection property. Since each $f$ is continuous, the sets $\operatorname{Fix}(f)$ are compact and therefore $M=\cap\{\operatorname{Fix}(f): f \in \mathcal{F}\}$ is nonempty and compact. As such, $M$ has a minimum element $a$ and a maximum element $b$; so $M \subset[a, b]$. But for $f \in \mathcal{F}, a$ and $b$ are fixed points of $f$; therefore, $[a, b] \subset \operatorname{Fix}(f)$ since $\operatorname{Fix}(f)$ is an interval. But then $[a, b] \subset M$ since $f$ was an arbitrary element of $\mathcal{F}$. We conclude, $M=[a, b]$.

Example 4.1. Let $E$ be the rectangle $[-1,1] \times[0,1]$ in the plane $\left(\mathbb{R}^{2}, d\right)$ and let $A=$ $(0,1), B=(1,0)$ and $C=(-1,0)$. Let $[A, B],[C, B]$, and $[C, A]$ denote the sides of the triangle $\mathrm{ABC}$. We shall use the "maximum" metric $d\left(\left(x_{1}, y_{1}\right),\left(x_{2}, y_{2}\right)\right)=\max \left\{\left|x_{1}-x_{2}\right|, \mid\right.$ $\left.y_{1}-y_{2} \mid\right\}$ so that $\mathbb{R}^{2}$ and therefore $E$ is hyperconvex. Now define $f: E \rightarrow[A, B]$ and $g$ : $E \rightarrow[C, A]$ as follows;

$f(g)$ sends the point $(c, d)$ of $E$ into the point of intersection of the line $y=d$ with the line segment $[A, B]([C, A])$. Define $h: E \rightarrow[C, B]$ by $h((c, d))=(c, 0)$. Then $f, g$ and $h$ are nonexpansive self-maps of $E$ having the segments $[A, B],[C, A]$, and $[C, B]$ as their respective fixed point sets. The point $A$ is the only point of coincidence, coincidence point, and fixed point of $f$ and $g$. Consequently, as is easy to show, $f$ and $g$ commute at A; i.e., $f$ and $g$ are nontrivially weakly compatible. Similarly, the pairs $\{g, h\}$ and $\{h, f\}$ have the points $C$ and $B$ as their respective unique common fixed points and are thus weakly compatible pairs. However, the family $\{f, g, h\}$ has no common fixed point. The functions $f, g, h$ clearly have no nontrivial periodic points. Consequently, the likelihood of extending Theorem 4.3 to hyperconvex spaces more general than $[0,1]$ is small. It should be noted that the pairs $\{f, g\},\{g, h\}$ and $\{h, f\}$ are all $R$-pairs, and that they provide examples satisfying the hypothesis of Theorem 3.1.

\section{Acknowledgements}

The authors would like to thank the referees for their comments that undoubtedly helped us to improve the text. The first author gratefully acknowledges the support from the Deanship of Scientific Research (DSR) at King Abdulaziz University (KAU) during this research.

\section{Author details}

${ }^{1}$ Department of Mathematics, King Abdulaziz University, P.O. Box 80203, Jeddah 21589, Saudi Arabia ${ }^{2}$ Department of Mathematics, Bradley University, Peoria, IL 61625, USA ${ }^{3}$ Department of Mathematical Sciences, The University of Texas at El Paso, El Paso, TX 79968, USA ${ }^{4}$ Department of Mathematics \& Statistics, King Fahd University of Petroleum and Minerals, Dhahran 31261, Saudi Arabia

\section{Authors' contributions}

All the authors contributed equally. All authors read and approved the final manuscript.

\section{Competing interests}

The authors declare that they have no competing interests.

Received: 6 March 2012 Accepted: 20 June 2012 Published: 20 June 2012

\section{References}

1. DeMarr, R: Common fixed points for commuting contraction mappings. Pac J Math. 13, 1139-1141 (1963)

2. Khamsi, MA, Lin, M, Sine, R: On the fixed points of commuting nonexpansive maps in hyperconvex spaces. J Math Anal Appl. 168, 372-380 (1992). doi:10.1016/0022-247X(92)90165-A 
3. Hussain, N, Khamsi, MA, Latif, A: Banach operator pairs and common fixed points in hyperconvex metric spaces. Nonlinear Anal. 74, 5956-5961 (2011). doi:10.1016/..na.2011.05.072

4. Aronszajn, N, Panitchpakdi, P: Extensions of uniformly continuous transformations and hyperconvex metric spaces. Pac J Math. 6, 405-439 (1956)

5. Chen, J, Li, Z: Banach operator pair and common fixed points for nonexpansive maps. Nonlinear Anal. 74, 3086-3090 (2011). doi:10.1016/j.na.2010.12.010

6. Hussain, N: Common fixed points in best approximation for Banach operator pairs with Ciric type 1 -contractions. J Math Anal Appl. 338, 1351-1362 (2008). doi:10.1016/j.jmaa.2007.06.008

7. Pathak, HK, Hussain, N: Common fixed points for Banach operator pairs with applications. Nonlinear Anal. 69 2788-2802 (2008). doi:10.1016/j.na.2007.08.051

8. Khan, AR, Akbar, F: Best simultaneous approximations, asymptotically nonexpansive mappings and variational inequalities in Banach spaces. J Math Anal Appl. 354, 469-477 (2009). doi:10.1016/j.jmaa.2009.01.007

9. Jungck, G: Common fixed points for commuting and compatible maps on compacta. Proc Am Math Soc. 103, 977-983 (1988). doi:10.1090/S0002-9939-1988-0947693-2

10. Jungck, G: Common fixed point theorems for compatible self maps of Hausdorff topological spaces. Fixed Point Theory Appl. 2005, 355-363 (2005)

11. Shimizu, T, Takahashi, W: Fixed point theorems in certain convex metric spaces. Math Jpn. 37, 855-859 (1992)

12. Espínola, R, Fernández-León, A, Piatek, B: Fixed points of single and set-valued mappings in uniformly convex metric spaces with no metric convexity. Fixed Point Theory Appl 16 (2010). Article ID 169837

13. Espínola, R, Lorenzo, P, Nicolae, A: Fixed points, selections and common fixed points for nonexpansive-type mappings. J Math Anal Appl. 382, 503-515 (2011). doi:10.1016/j.jmaa.2010.06.039

14. Suzuki, T: Fixed point theorems and convergence theorems for some generalized nonexpansive mappings. J Math Anal Appl. 340, 1088-1095 (2008). doi:10.1016/j.jmaa.2007.09.023

15. Hussain, N, Khamsi, MA: On asymptotic pointwise contractions in metric spaces. Nonlinear Anal Theory Methods Appl. 71, 4423-4429 (2009). doi:10.1016/j.na.2009.02.126

16. Jungck, G: Commuting mappings and fixed points. Am Math Mon. 83, 261-263 (1976). doi:10.2307/2318216

17. Abbas, $\mathrm{M}$, Jungck, $\mathrm{G}$ : Common fixed point results for noncommuting mappings without continuity in cone metric spaces. J Math Anal Appl. 341, 416-420 (2008). doi:10.1016/j.jmaa.2007.09.070

18. Jungck, G, Hussain, N: Compatible maps and invariant approximations. J Math Anal Appl. 325, 1003-1012 (2007). doi:10.1016/j.jmaa.2006.02.058

19. Espínola, R, Hussain, N: Common fixed points for multimaps in metric spaces. Fixed Point Theory Appl 2010, 14 (2010). Article ID 204981

20. Hussain, N, Khamsi, MA, Kirk, WA: One-local retracts and Banach operator pairs in metric spaces. Appl Math Comput. 218, 10072-10081 (2012). doi:10.1016/j.amc.2012.03.067

21. Kirk, WA: Geodesic geometry and fixed point theory II. International Conference on Fixed Point Theory and Applications. pp. 113-142.Yokohama Publishers, Yokohama (2004)

22. Kirk, WA: Universal nonexpansive maps, fixed point theory and its applications. pp. 95-101. Yokohama Publishers, Yokohama (2008)

23. Jungck, G: Common fixed points for compatible maps on the unit interval. Proc Am Math Soc. 115, 495-499 (1992) doi:10.1090/S0002-9939-1992-1105040-0

doi:10.1186/1687-1812-2012-100

Cite this article as: Hussain et al: Nonexpansive retracts and weak compatible pairs in metric Spaces. Fixed Point

Theory and Applications 2012 2012:100.

\section{Submit your manuscript to a SpringerOpen ${ }^{\circ}$ journal and benefit from:}

- Convenient online submission

- Rigorous peer review

- Immediate publication on acceptance

- Open access: articles freely available online

- High visibility within the field

- Retaining the copyright to your article

Submit your next manuscript at $\boldsymbol{s p r i n g e r o p e n . c o m ~}$ 